\title{
Identification of differentially expressed proteins and clinicopathological significance of HMGB2 in cervical cancer
}

\author{
Xiao Han ${ }^{1,2+}$, Siyi Zhong ${ }^{1,2+}$, Pengnan Zhang ${ }^{2,3}$, Yanmei Liu ${ }^{1,2}$, Sangsang Shi ${ }^{1,2}$, Congquan Wu $\mathrm{W}^{1,2}$ \\ and Shujun Gao ${ }^{1,2^{*}}$
}

\begin{abstract}
To investigate the complexity of proteomics in cervical cancer tissues, we used isobaric tags for relative and absolute quantitation (iTRAQ)-based mass spectrometry analysis on a panel of normal cervical tissues (N), high-grade squamous intraepithelial lesion tissues (HSIL) and cervical cancer tissues (CC). Total 72 differentially expressed proteins were identified both in CC vs N and CC vs HSIL. The expression of HMGB2 was markedly higher in CC than that in HSIL and N. High HMGB2 expression was significantly correlated with primary tumor size, invasion and tumor stage. The up-regulated HMGB2 was discovered to be associated with human cervical cancer. These findings suggest that HMGB2 may be a potentially prognostic biomarker and a target for the therapy of cervical cancer.
\end{abstract}

Keywords: Cervical cancer, Proteomics, HMGB2, Tumor progression

\section{Introduction}

Cervical cancer is one of the most frequent malignant tumors and is the main cause of cancer-related mortality of women worldwide every year $[1,2]$. Although the survival rates of the cancer patients have been improved, the advance in cervical cancer doesn't match those observed in other common tumors, partly because of that cervical cancer patients were often diagnosed in an advanced stage [3]. Recently, the advances in biological behavior and molecular pathogenesis of cancers have resulted in the progression of molecular targeted therapies [4]. However, effective drug targets are limited. Thus, further research on molecular mechanisms underlying cervical cancer development is much needed to identify new therapeutic targets.

*Correspondence: 031108282@fudan.edu.cn

${ }^{\dagger}$ Xiao Han and Siyi Zhong contributed equally to this work

${ }^{1}$ Center of Diagnosis and Treatment for Cervical Diseases, Obstetrics

and Gynecology Hospital of Fudan University, No. 419, Fangxie Road,

Huangpu District, Shanghai 200011, China

Full list of author information is available at the end of the article
The molecular features of cancer are fiendishly complex because of cancer heterogeneity. As proteins are the primary functional macromolecule in organisms, proteomics attracted the public attention and are applied for various types of cancers. For example, by MS-based quantitative proteomic analysis, Moon-Won Yoo et al. identified and verified that four proteins were useful for discriminate patients with gastric cancer from normal persons [5]. Swiatly et al. highlighted several dysregulated proteins related to ovarian cancer by isobaric tags for relative and absolute quantification (iTRAQ)-based mass spectrometry analysis, in which five proteins were validated to differentially express in ovarian cancer tissues and could improve diagnostic performance [6]. For hepatocellular carcinoma research, 52 proteins were identified to interact with $C$ reactive protein by iTRAQ-based proteomic profiling, contributing to the understanding of molecular pathogenesis of hepatocellular carcinoma [7]. All these researches showed the vital role of proteomics in the field of cancer investigation.

However, despite a proteomics analysis of serum samples from patients with cervical cancer and cervical

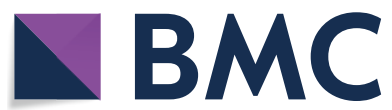

(c) The Author(s) 2020. This article is licensed under a Creative Commons Attribution 4.0 International License, which permits use, sharing, adaptation, distribution and reproduction in any medium or format, as long as you give appropriate credit to the original author(s) and the source, provide a link to the Creative Commons licence, and indicate if changes were made. The images or other third party material in this article are included in the article's Creative Commons licence, unless indicated otherwise in a credit line to the material. If material is not included in the article's Creative Commons licence and your intended use is not permitted by statutory regulation or exceeds the permitted use, you will need to obtain permission directly from the copyright holder. To view a copy of this licence, visit http://creativeco mmons.org/licenses/by/4.0/. The Creative Commons Public Domain Dedication waiver (http://creativecommons.org/publicdomain/ zero/1.0/) applies to the data made available in this article, unless otherwise stated in a credit line to the data. 
intraepithelial neoplasis [8], there are few researches on dysregulated proteins analysis for cervical cancer tissue samples. Considering that several factors such as sample type and study design would affect the amount of proteomic studies data for cancer [9], we identified differentially expressed proteins in cervical cancer samples compared to high-grade squamous intraepithelial lesion samples and normal cervical samples by a particularly powerful tool iTRAQ-based mass spectrometry. Function annotation and protein-protein interaction network analysis of the identified proteins were carried out. Moreover, the highlighted protein was validated by immunohistochemistry staining; its clinical significance was assessed in cervical cancer patients.

\section{Materials and methods}

\section{Patients and samples}

Normal cervical tissues $(\mathrm{N}, \mathrm{n}=27)$, high-grade squamous intraepithelial lesion tissues (HSIL, $n=24$ ) and cervical squamous cell cancer tissues $(C C, n=29)$ were obtained by colposcopy biopsy in the Obstetrics and Gynecology Hospital affiliated with Fudan University, China from $12 / 26 / 2014$ to $12 / 25 / 2016$. The HPV information of the samples was showed in Additional file 1: Table S1. The tumor stage of cervical cancer was categorized according to surgical and pathological findings, basing on the guidelines described by the International Federation of Gynecology and Obstetrics (FIGO) stage system. This research was approved by the review board and ethics committee of Obstetrics and Gynecology Hospital affiliated with Fudan University. Patients without any preoperative therapy were included. Written informed consents were obtained from all patients. For the iTRAQ-based mass spectrometry analysis, total 8 samples from CC, HSIL and $\mathrm{N}$ were selected, respectively. For the immunohistochemistry staining, all samples of the three groups were used.

\section{ITRAQ labeling, strongcatiobexchange fractionation and LC-MS/MS analysis}

The protein concentration was detected with the $\mathrm{BCA}$ method. The iTRAQ labeling and strong catiob-exchange fractionation were performed according to the reported methods [10]. Firstly, $200 \mu \mathrm{g}$ protein of each group was used for iTRAQ labeling, which was performed with tris (2-carboxyethyl) phosphine (TCEP) for $1 \mathrm{~h}$ of reduction at $60{ }^{\circ} \mathrm{C}$ and then with S-methyl methanethiosulfonate (MMTS) for $20 \mathrm{~min}$ of alkylation at room temperature. Subsequently, after $16 \mathrm{~h}$ of digestion at $37^{\circ} \mathrm{C}$ using sequencing grade trypsin (Promega, San Luis Obispo, CA) (enzyme/protein (mass ration) $=1: 20$ ), the digestion production was separately labeled with 8-plex iTRAQ reagents (AB SCIEX, Washington, D.C.). After reconstituted with Solvent A (25\% acetonitrile, $10 \mathrm{mM}$ $\mathrm{KH}_{2} \mathrm{PO}_{4}, \mathrm{pH} 2.8$ ), the samples were fractionated in the PolySULFOETHYL A column $(200 \times 4.6 \mathrm{~mm}, 5 \mu \mathrm{m}$, $200 \AA$ Å, PolyLC Inc., Columbia, MD) with an Agilent 1260 series high performance liquid chromatography system. Peptides were fractionated more than 50 min with a constant velocity $(350 \mu \mathrm{l} / \mathrm{min})$ by a concentration gradient from 0 to $60 \%$ solvent B $(10 \mathrm{mM}, 25 \%$ acetonitrile and $350 \mathrm{mM} \mathrm{KCl}, \mathrm{pH} 2.8$ ). The fractions were collected and pooled into 20 fractions, followed by reconstituted with formic acid $(0.1 \%)$ and desalted with C18 StageTips (3 M Empore, St. Paul, MN). LC-MS/MS analysis for the samples was performed on the LTQ-Orbitrap Velos mass spectrometer (Thermo Scientific, Bremen, Germany) with an instrument interface of Easy-nLC II system (Thermo Scientific, Bremen, Germany).

\section{Protein quantification and identification}

Proteome Discoverer software (version 1.4.0.288; Thermo Fisher Scientific) was used to extract tandem mass spectra without performing charge state deconvolution and deisotoping. All MS/MS samples were analyzed with Mascot software (version 1.4.0.288; Matrix Science, London, UK) and Sequest software (version 1.4.0.288; Thermo Fisher Scientific, San Jose, CA, USA). Mascot and Sequest were separately set up to search SwissProt database (Homo sapiens, 20411 entries) with trypsin as the digestion enzyme. Mascot was searched with a fragment ion mass tolerance of 20 PPM and a parent ion tolerance of 10.0 PPM, while Sequest was searched with the same parent ion tolerance and different fragment ion mass tolerance $(0.020 \mathrm{Da})$. Carbamidomethyl of cysteine and iTRAQ8plex of lysine and the n-terminus were specified as fixed modifications in both Mascot and Sequest. The variable modifications (oxidation of methionine and iTRAQ8plex of tyrosine) were also specified in both Mascot and Sequest. MS/MS based peptide and protein identifications were validated using Scaffold (version Scaffold_4.0.5, Proteome Software Inc., Portland, OR). If a probability of more than $99.0 \%$ to achieve a false discovery rate (FDR) less than $1.0 \%$ was established by the Scaffold Local FDR algorithm, the peptide identifications were accepted. While a probability of more than $99.0 \%$ to achieve a FDR less than $1.0 \%$, the protein identifications were accepted. All the proteins identified contained at least 7 amino acids and at least 1 unique peptide. Protein Prophet algorithm was used to assign the protein probabilities. To meet the principles of parsimony, the proteins containing similar peptides, which couldn't be differentiated by MS/MS analysis alone, were grouped. Matrix correction of channels in all samples were performed according to the reported i-Tracker algorithm [11]. The obtained intensity was globally normalized 
within all acquisition runs. Each quantitative sample was normalized in each acquisition run. The identification intensity of each peptide was normalized within the specified protein. The referenced channels were standardized to achieve a fold change of 1:1. All normalization computations used the medians to multiply and normalize the data. Mann Whitney Test analysis was performed to determine the differentially expressed proteins. Fold change (FC) of the proteins was calculated for the comparisons of CC vs $\mathrm{N}$ and CC vs HSIL, respectively. The proteins with a threshold of $\left|-\log _{2} \mathrm{FC}\right| \geq 1.0$ and $p$ value $<0.05$ were identified as significantly differentially expressed proteins.

\section{Bioinformatics analysis}

The overlapped proteins of the significantly differentially expressed proteins in CC vs $\mathrm{N}$ and CC vs HSIL were selected for function annotation and protein-protein interaction (PPI) network analysis. The function annotation was carried out with DAVID Bioinformatics Resources (version 6.8; https://david.ncifcrf.gov/) [12, 13] and PANTHER Classification System (version 14.0; http://www.pantherdb.org/) [14], respectively. The PPI network analysis was carried out with STRING (version 11.0; https://string-db.org/) and the network was graphed with the Cytoscape software (version 3.6.0) $[15,16]$. The sub-networks were identified using the MCODE plugin in Cytoscape with the default parameters.

\section{Immunohistochemistry staining}

Paraffin-embedding tissues were cut to a thickness of $4 \mu \mathrm{m}$ slides. After $10 \mathrm{~min}$ of antigen retrieval by the microwave oven at $95{ }^{\circ} \mathrm{C}$, the slides were incubated with the primary antibody anti-HMGB2 (Ab124670, Abcam). The negative control slides were incubated with normal mouse IgG. The slides were rinsed with phosphate-buffered saline for several times and then stained with the Elivision TM Plus Polymer HRP (Mouse/Rabbit) IHC kit (Maixin Biological Technology Development Co., Fuzhou, China), which is based on the visualization of streptavidin-biotin-peroxidase. The slides were incubated with biotin-conjugated IgG (goat anti-mouse polyclonal antibody) for $20 \mathrm{~min}$ at $25^{\circ} \mathrm{C}$, and subsequently incubated with a streptavidin-biotin-HRP complex at $25{ }^{\circ} \mathrm{C}$ for $20 \mathrm{~min}$. After washing, the slides were visualized via incubating with 3,3-diaminobenzidine solution (Maixin Biotechnology Development Co., Ltd). The nuclei were stained with hematoxylin.

\section{Assessment of immunohistochemical staining}

The expression of HMGB2 expression was assessed semiquantitatively based on immunohistochemical staining. The HMGB2 protein expression was separately evaluated by 2 blinded observers. Each slide obtained an immunoreactive score (IRS; 3 , strong; 2, moderate; 1 , weak; 0 , negative) according to nuclear staining intensity. Cut point for high HMGB2 expression or positive: IRS $\geq 2$; Cut point for low HMGB2 expression or negative: IRS $<2$.

\section{Statistical analysis}

SPSS (version 19.0, Chicago, IL, USA) was used for statistical analysis. Data were expressed as mean \pm standard error of the mean (SEM). Pearson's chi-squared test and the Student two-sided $t$ test were used to analyze the correlation between the expression of HMGB2 and clinicopathological factors. $\mathrm{P}<0.05$ was considered statistically significant.

\section{Results}

\section{Identification of differentially expressed proteins}

To characterize proteomic alterations in the primary cervical cancer samples, an iTRAQ-based mass spectrometry analysis approach was employed. Total 5473 proteins were identified in all the samples. Dysregulated proteins were quantified basing on the iTRAQ labels intensity for unique peptides. Total 91 significantly differentially expressed proteins were identified between cervical cancer samples and normal cervical samples (CC vs N); total 106 significantly differentially expressed proteins were identified between cervical cancer tissues and high-grade squamous intraepithelial lesion tissues (CC vs HSIL) (Fig. 1a). Among these proteins, 73 proteins were overlapped in the comparisons of $\mathrm{CC}$ vs $\mathrm{N}$ and $\mathrm{CC}$ vs HSIL (Fig. 1b). Of the 73 proteins, 72 proteins with the consistent regulatory relationships, namely simultaneous up-regulation or simultaneous down-regulation in CC vs $\mathrm{N}$ and CC vs HSIL, were selected for further analysis. The heatmap of 72 identified proteins suggested that three different groups (CC, N and HSIL) were distinguished by clustering (Fig. 1c).

\section{Function annotation of identified proteins}

Based on the PANTHER Classification System, 72 identified proteins were categorized into 18 protein classes (Fig. 2a). Nucleic acid binding (22.2\%), oxidoreductase (11.1\%), signaling molecule (8.9\%) and transcription factor $(8.9 \%)$ were the four largest classes. Gene ontology (GO) analysis of 72 identified proteins was performed on both PANTHER and DAVID from three aspects: molecular function, cellular component and biological process. The top 10 enriched GO terms by DAVID were showed in Fig. 2b; the mainly enriched GO terms by PANTHER were showed in Fig. 2c. Both the GO results of PANTHER and DAVID showed that the identified proteins were mainly related to binding and structural molecule activity, involved in biological regulation and various 
a

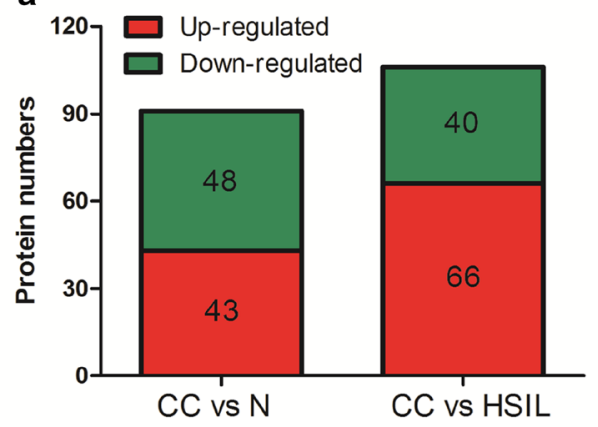

b CC vs $\mathrm{N}$ (up)

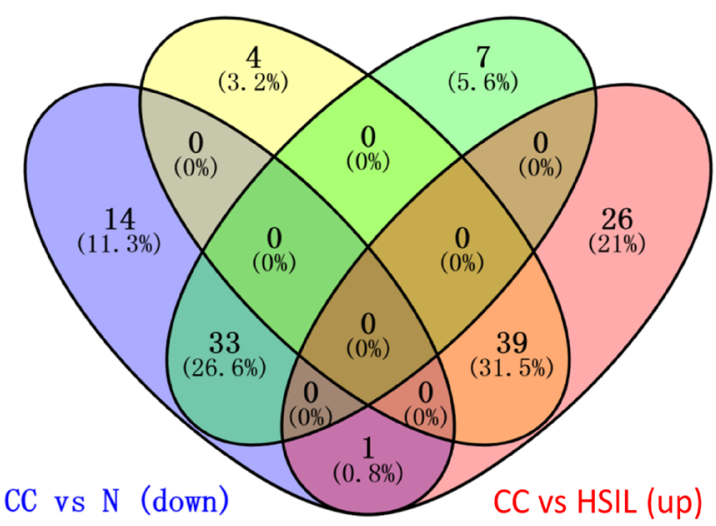

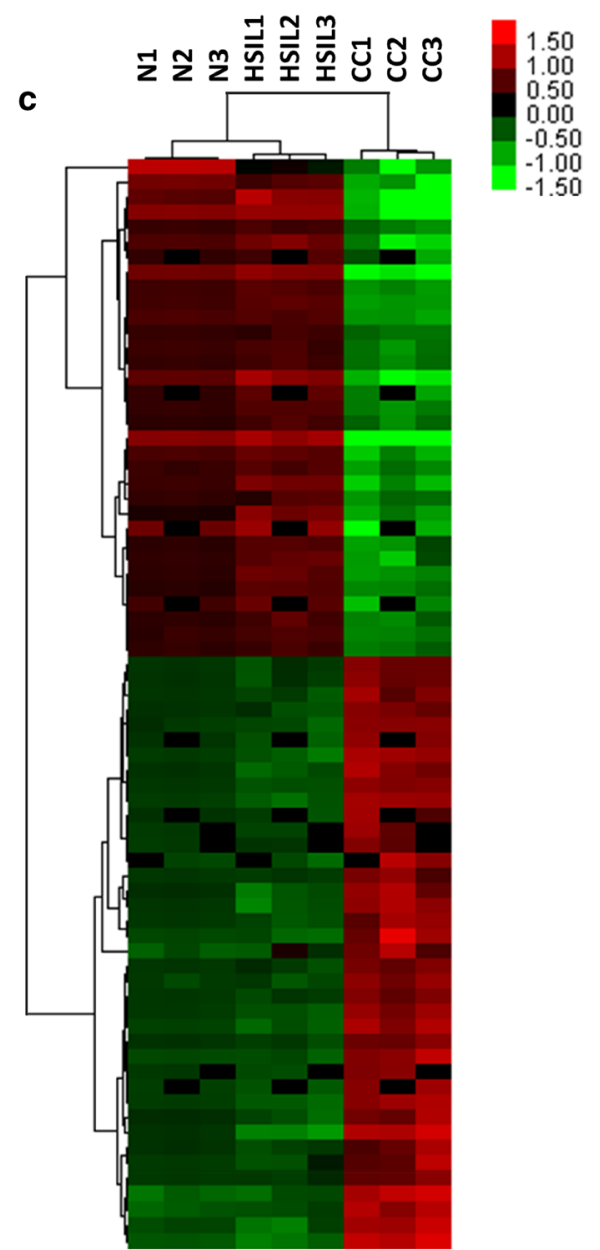

Fig. 1 Identification of significantly differentially expressed proteins. a The protein numbers of significantly differentially expressed proteins in cervical cancer tissues (CC) compared to normal cervical tissues (N) and high-grade squamous intraepithelial lesion tissues (HSIL). b The overlapped proteins in the comparisons of CC vs N and CC vs HSIL. c The heatmap of overlap proteins. $n=3$ indicate the three repeats from the pooled samples of each group

immune-related biological process (antigen processing and presentation, inflammatory response to antigenic stimulus, defense response to virus, response to stimulus, immune system process, etc.), and were presented in extracellular region, membrane and protein complex. The top $10 \mathrm{GO}$ terms (biological process and molecular function) and involved proteins analyzed by DAVID are shown in Table 1.

\section{PPI network analysis of identified proteins}

PPI network analysis was performed using STRING. Three clusters were identified by sub-networks analysis using MCODE plugin in the Cytoscape software (Fig. 3). The cluster 1 was mainly composed of HMG box transcription factor chromatin and chromatin-binding protein signaling molecule (HMGB1 and HMGB2), and histone (H1F0, HIST1H1C, HIST1H1D and HIST1H1E).
The cluster 2 was made up of HLA-DRA, HLA-B, HLADRB1, HLA-C, GBP1, ISG20 and IFIT1. Among these proteins, HLA-DRA and HLA-DRB1 was belonged to major histocompatibility complex antigen protein class. The cluster 3 contained tubulin TUBB4B. Here, we reported that the up-regulated HMGB2 was associated with human cervical cancer. Thus, the expression of HMGB2 was validated and its clinical significance was studied further.

\section{Immunohistochemical expression of HMGB2}

The expression of HMGB2 was determined by immunohistochemistry staining. There were no tissues represented negative immunohistochemical staining for HMGB2. The representative microphotographs of IRS score 1, 2 and 3 were shown in Additional file 1: Figure $\mathrm{S} 1$. As shown in Fig. 4a-f, immunohistochemistry stains 
a

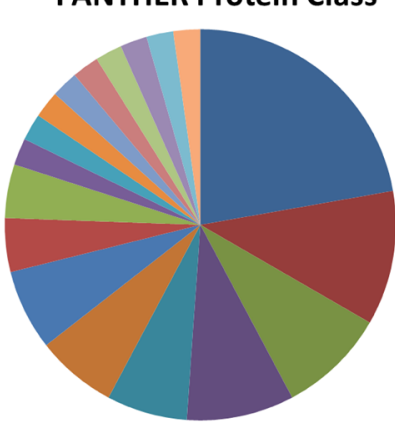

nucleic acid binding (22.2\%)

- oxidoreductase (11.1\%)

n signaling molecule (8.9\%)

- transcription factor (8.9\%)

n cytoskeletal protein $(6.7 \%)$

nenzyme modulator (6.7\%)

defense/immunity protein (6.7\%)

calcium-binding protein $(4.4 \%)$

nydrolase (4.4\%)

- extracellular matrix protein (2.2\%)

- transporter (2.2\%)

- transferase $(2.2 \%)$

cell adhesion molecule (2.2\%)

- ligase (2.2\%)

- transfer/carrier protein (2.2\%)

membrane traffic protein (2.2\%)

nstructural protein (2.2\%)

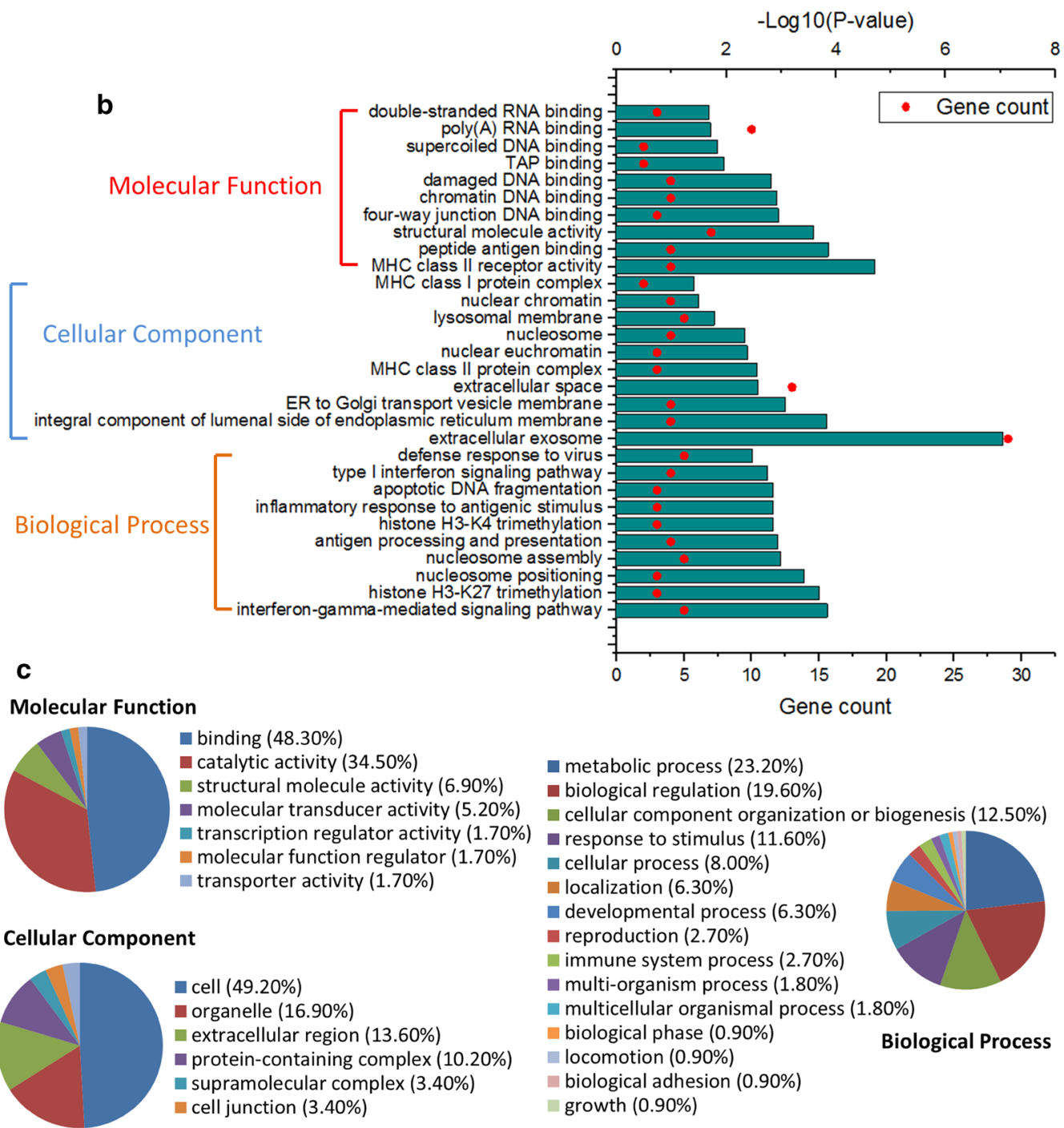

Fig. 2 The function annotation of overlapped proteins was analyzed based on PANTHER Classification System and DAVID Bioinformatics Resources. a The PANTHER protein class of the overlapped proteins. The gene ontology analysis of overlapped proteins by DAVID (b) and PANTHER (c) 
Table 1 Top $10 \mathrm{GO}$ terms (biological process and molecular function) and involved proteins by DAVID

\begin{tabular}{|c|c|c|c|c|}
\hline GO ID & Term & $P$ value & Involved proteins & Fold Enrichment \\
\hline \multicolumn{5}{|c|}{ Biological process } \\
\hline GO:0060333 & Interferon-gamma-mediated signaling pathway & $1.45 \times 10^{-4}$ & HLA-DRB1, HLA-C, HLA-B, HLA-DRA, GBP1 & 18.48 \\
\hline GO:0098532 & Histone H3-K27 trimethylation & $2.06 \times 10^{-4}$ & HIST1H1E, HIST1H1D, HIST1H1C & 131.19 \\
\hline GO:0016584 & Nucleosome positioning & $3.82 \times 10^{-4}$ & HIST1H1E, HIST1H1D, HIST1H1C & 98.39 \\
\hline GO:0006334 & Nucleosome assembly & $1.03 \times 10^{-3}$ & H1F0, HIST1H1E, HMGB2, HIST1H1D, IST1H1C & 11.02 \\
\hline GO:0019882 & Antigen processing and presentation & $1.14 \times 10^{-3}$ & HLA-DRB1, HLA-C, HLA-B, HLA-DRA & 19.09 \\
\hline GO:0080182 & Histone $\mathrm{H} 3-\mathrm{K} 4$ trimethylation & $1.41 \times 10^{-3}$ & HIST1H1E, HIST1H1D, HIST1H1C & 52.48 \\
\hline GO:0002437 & Inflammatory response to antigenic stimulus & $1.41 \times 10^{-3}$ & HMGB1, HMGB2, HLA-DRB1 & 52.48 \\
\hline GO:0006309 & Apoptotic DNA fragmentation & $1.41 \times 10^{-3}$ & H1FO, HMGB1, HMGB2 & 52.48 \\
\hline GO:0060337 & Type I interferon signaling pathway & $1.78 \times 10^{-3}$ & IFIT1, HLA-C, HLA-B, ISG20 & 16.40 \\
\hline GO:0051607 & Defense response to virus & $3.41 \times 10^{-3}$ & AZU1, IFIT1, DEFA3, ISG20, GBP1 & 7.96 \\
\hline \multicolumn{5}{|c|}{ Molecular function } \\
\hline GO:0032395 & MHC class II receptor activity & $1.98 \times 10^{-5}$ & KRT17, HLA-DRB1, HLA-C, HLA-DRA & 72.61 \\
\hline GO:0042605 & Peptide antigen binding & $1.38 \times 10^{-4}$ & HLA-DRB1, HLA-C, HLA-B, HLA-DRA & 38.90 \\
\hline GO:0005198 & Structural molecule activity & $2.62 \times 10^{-3}$ & KRT6C, PGM5, KRT17, FLG, SPRR1B, KRT1, SPRR3 & 7.72 \\
\hline GO:0000400 & Four-way junction DNA binding & $1.14 \times 10^{-3}$ & HMGB1, MSH6, HMGB2 & 58.34 \\
\hline GO:0031490 & Chromatin DNA binding & $1.20 \times 10^{-3}$ & H1F0, HIST1H1E, HIST1H1D, HIST1H1C & 18.78 \\
\hline GO:0003684 & Damaged DNA binding & $1.53 \times 10^{-3}$ & HMGB1, MSH6, HMGB2, MSH5 & 17.29 \\
\hline GO:0046977 & TAP binding & $1.08 \times 10^{-2}$ & $\mathrm{HLA}-\mathrm{C}, \mathrm{HLA}-\mathrm{B}$ & 181.52 \\
\hline GO:097100 & Supercoiled DNA binding & $1.44 \times 10^{-2}$ & HMGB1, HMGB2 & 136.14 \\
\hline GO:0044822 & Poly(A) RNA binding & $1.94 \times 10^{-2}$ & $\begin{array}{l}\text { H1F0, HMGB1, HIST1H1E, HMGB2, HIST1H1D, } \\
\text { HIST1H1C, TRA2B, RPL26, TMSB4X, MANF }\end{array}$ & 2.41 \\
\hline GO:0003725 & Double-stranded RNA binding & $2.05 \times 10^{-2}$ & HMGB1, YRDC, TUBB4B & 13.39 \\
\hline
\end{tabular}

of HMGB2 in almost all the samples were in nucleus. Strong HMGB2 immunoreactivity (20/29) was found in cervical cancer tissues (Fig. 4a, b). Most of high-grade squamous intraepithelial lesion tissues (HSIL) had weak nuclear stains for HMGB2 (Fig. 4c, d). Only 4 of 24 HSIL samples showed moderate immunoreactivity of HMGB2. Almost no cases showed HMGB2 immunoreactivity in those normal cervical tissues (Fig. 4e, f). Overall, the expression of HMGB2 in cervical cancer samples was significantly higher than in HSIL and normal cervical samples (both $P<0.05$; Fig. $4 \mathrm{~g}$ ). Interestingly, positive reaction of HMGB2 was gradually higher during the progression of cervical cancer.

\section{HMGB2 expression in was associated with clinicopathological factors}

To investigate the correlations between HMGB2 expression and clinicopathological factors in cervical cancer, the expression level of HMGB2 was represented with an immunoreactive scored (IRS; 3 , strong; 2 , moderate; 1 , weak; 0, negative). Of 29 cervical cancer samples, $9(35.5 \%)$ samples with IRS of 0-1 were classified as low HMGB2 expression (HMGB2-low), and 20 (64.5\%) samples with IRS of 2-3 were classified as high HMGB2 expression (HMGB2-high). Table 2 showed the correlation analysis between HMGB2 expression and clinicopathological parameters of cervical cancer patients. The expression of HMGB2 was significantly associated with primary tumor size, invasion (infiltration depth) and FIGO stage $(P<0.05$, respectively).

\section{Discussion}

Dysregulated-proteins identification is of great significance for the discovery of biomarker, which can contribute to the early detection, therapeutic intervention and disease prognosis. In this study, total 72 differentially expressed proteins were identified to up-regulate in cervical cancer samples compared to both high-grade squamous intraepithelial lesion samples and normal cervical samples. Most of the 72 identified proteins were categorized into the nucleic acid binding (22.2\%) of PANTHER protein class. Gene ontology (GO) analysis results revealed that identified proteins were mainly related to binding and structural molecule activity, involved in biological regulation and various immune-related biological process, and were presented in extracellular region, membrane and protein complex. The results of proteinprotein interaction network analysis identified three clusters of highlighted proteins. Among these highlighted proteins, the members of HMG box transcription factor 


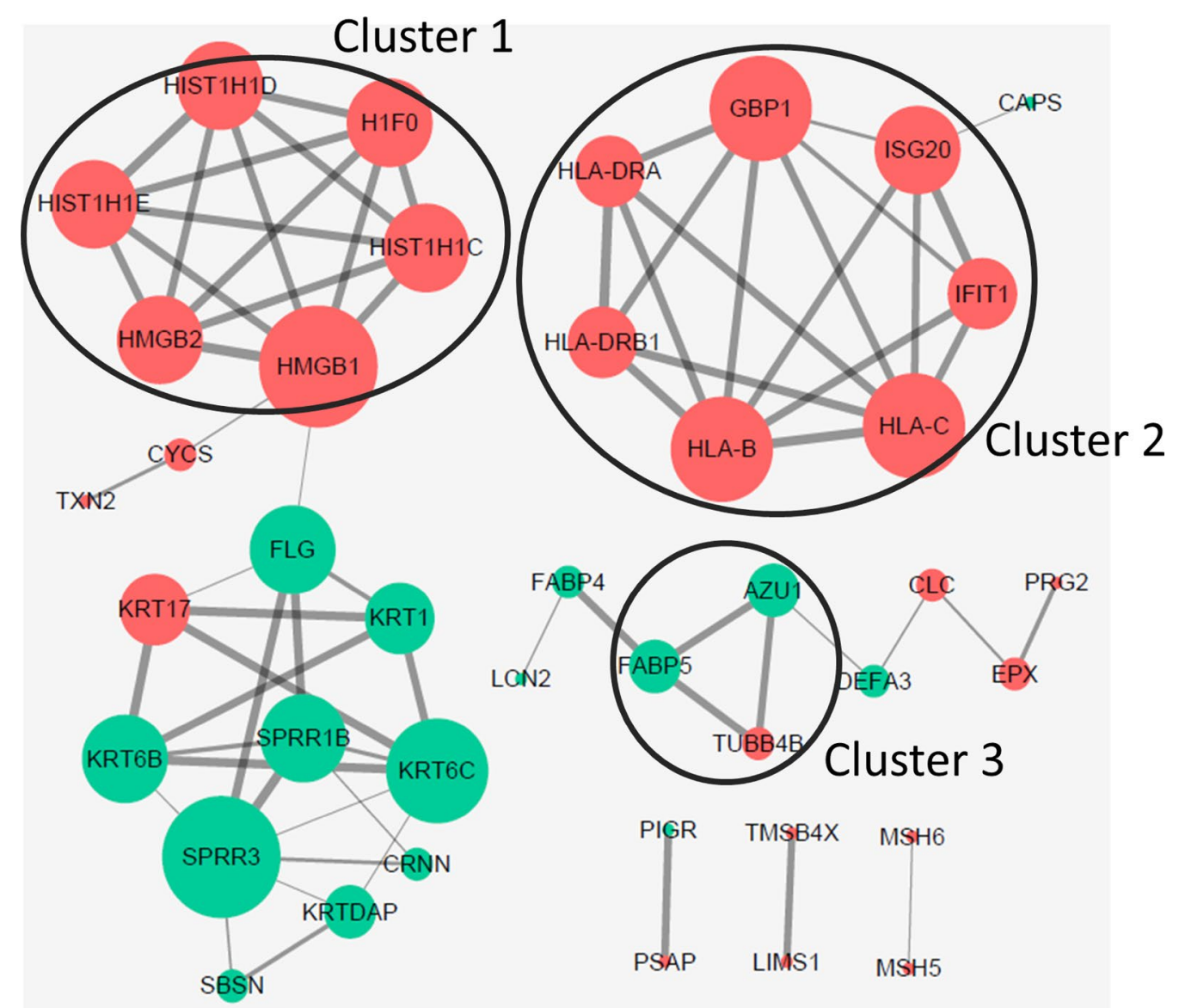

Fig. 3 The protein-protein network analysis of overlapped proteins based on STRING online database. Cluster 1, 2 and 3: the sub-networks identified by MCODE plugin in the Cytoscape. The red and green indicate to significantly up-regulated and down-regulated proteins in cervical cancer samples compared to both high-grade squamous intraepithelial lesion samples and normal cervical samples, respectively

chromatin and chromatin-binding protein signaling molecule (HMGB1 and HMGB2) attracted our attention. Here, we reported that the up-regulated HMGB2 was associated with human cervical cancer.

As ubiquitous and abundant nuclear non-histone chromosomal proteins, high-mobility group box (HMGB) proteins play an important role in binding to distorted DNA structures and subsequently regulating its transcription, replication, repair and recombination [17]. The HMGB family is comprised with HMGB1, HMGB2, HMGB3 and HMGB4. HMGB1 and HMGB2, which have greater than $80 \%$ identity of amino acid, are highly conserved with indistinguishable biological properties, including binding to DNA without specificity of the sequence [18]. It has been reported that HMGB1 is associated with a variety of diseases, including sepsis, arthritis and cancer [19-21]. Overexpression of HMGB1 has been found in numerous human cancers, such as pancreatic cancer [22], prostate cancer [23], breast cancer [24, 25], melanoma [26], colorectal cancer [27] and leukemia
[28]. More importantly, HMGB proteins preferred to bind to mis-incorporated nucleoside analogues or to cisplatinum (II) diamine dichloride (cisplatin)-modified DNA and subsequently inhibited the excision repair of nucleotide, which could have important value for cancer therapy [29-31]. Notably, HMGB1 expression has been reported to be related to tumor stage, invasion and metastasis in cervical squamous cell carcinoma as early as in year 2008 [32].

Considering the high homology of HMGB2 compared to HMGB1, it might have a similar role in the development of cancer. Koon et al. reported that HMGB2 was overexpressed in the malignant gastrointestinal stromal tumors and might be associated with the malignant behavior of gastrointestinal stromal tumors [33]. Then, overexpression of HMGB2 has been found in different kinds of human cancers, such as skin cancer [34], glioblastoma [35], hepatocellular carcinoma [36] and pancreatic cancer [37]. Lately, HMGB2 has been found to overexpress and promote cell proliferation 

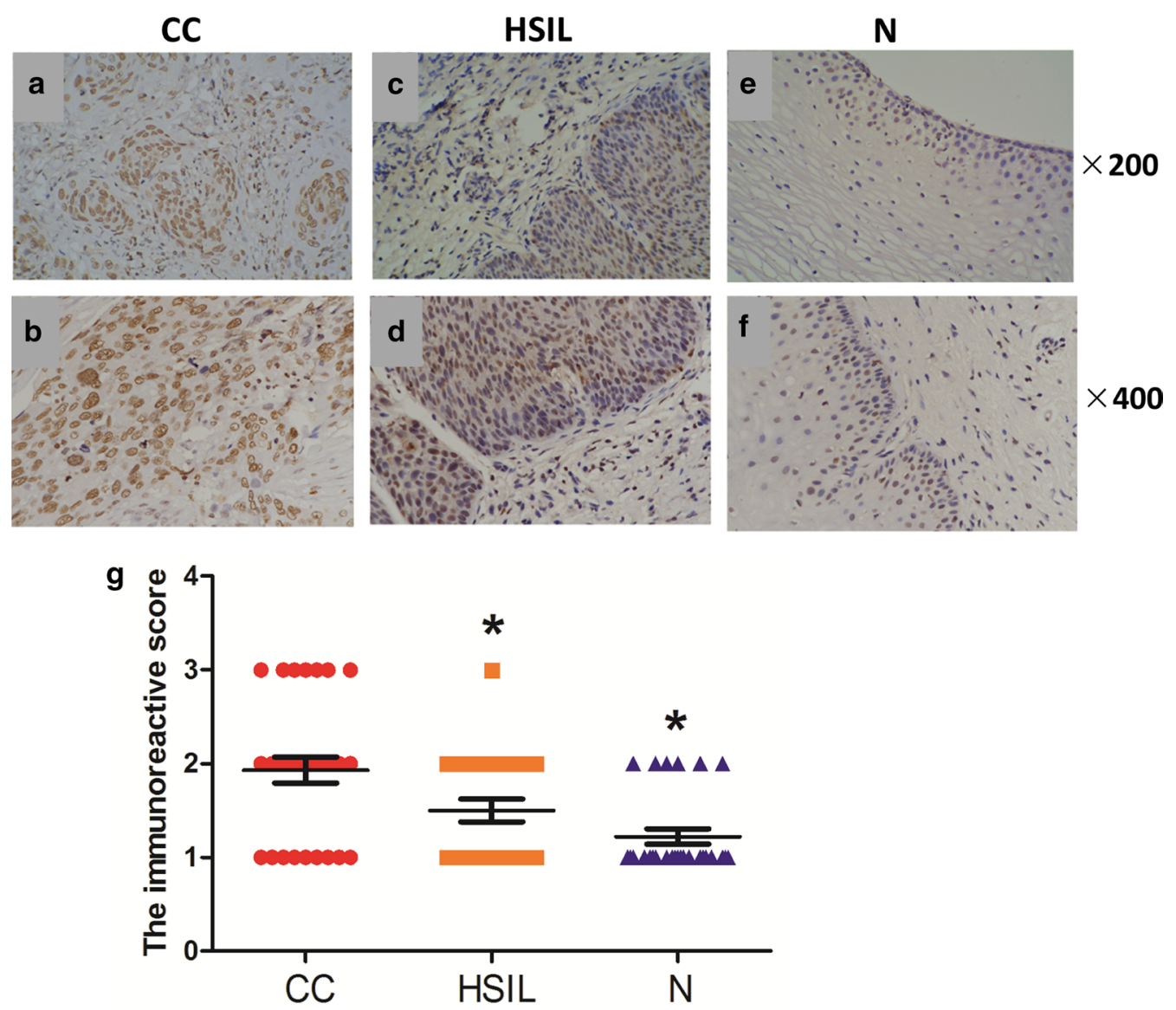

Fig. 4 Immunohistochemical expression of HMGB2 in cervical cancer samples (CC), normal cervical samples (N) and high-grade squamous intraepithelial lesion samples (HSIL). a-f Representative immunohistochemical staining images for CC, $N$ and HSIL. $\mathbf{g}$ The immunoreactive score for CC, N and HSIL. ${ }^{*} P<00.05$ compared to CC

and radiosensitivity through retinoblastoma-interaction-dependent or independent mechanisms [38]. Our recent research revealed that, according to the RNA-Seq data from The Cancer Genome Atlas (TCGA) program and The Genotype-Tissue Expression (GTEx) project, HMGB2 expression was significantly higher in cervical squamous cell carcinoma and endocervical adenocarcinoma than that in the normal controls; up-regulated HMGB2 expression promoted cell proliferation by activating AKT signaling pathway in cervical cancer cell lines [39]. Additionally, high expression of HMGB2 was associated with a poor prognosis for the patients with breast cancer via promoting cell proliferation and glycolysis in breast cancer cells [40, 41]. However, the expression and function of HMGB2, especially its relevance in carcinogenesis in cervical cancer remains largely unknown. Therefore, we detected the expression of HMGB2 in cervical cancer tissues by immunohistochemistry and evaluated the significance of HMGB2 expression in the clinical further. Here, our findings indicated that high expression of HMGB2 was significantly associated with primary tumor size, invasion (infiltration depth) and FIGO stage in cervical cancer. Although the exactly underlying mechanism of HMGB in prognosis of patients with cervical cancer remained uncovered, our results revealed that HMGB2 might at least partly promote cervical cancer progression and it might be a potentially prognostic biomarker for cervical cancer patients. These findings might facilitate future researches on the function of HMGB2 in cervical cancer.

Certainly, there are some limitations in this work. A limitation is that the small sample size. Eight samples of each group were randomly selected and then analyzed by iTRAQ 8 labeling and MS/MS. The way in which the samples were selected might resulted in some errors, and if the other different samples were selected, the estimation of FDR using sample permutation analysis might produce another different result. Notably, the result of immunohistochemical staining was consistent with that of proteomics analysis and thus enhanced the credibility. 
Table 2 Correlations of HMGB2 expression with clinicopathological parameters in primary cervical cancer tissues

\begin{tabular}{|c|c|c|c|c|}
\hline \multirow[t]{2}{*}{ Variables } & \multirow[t]{2}{*}{ No. of cases } & \multicolumn{2}{|c|}{$\begin{array}{l}\text { HMGB2 } \\
\text { expression }\end{array}$} & \multirow[t]{2}{*}{$P$ value } \\
\hline & & Low & High & \\
\hline \multicolumn{5}{|l|}{ Age (year) } \\
\hline$<50$ & 15 & 4 & 11 & \multirow[t]{2}{*}{0.273} \\
\hline$\geq 50$ & 14 & 5 & 9 & \\
\hline \multicolumn{5}{|c|}{ Family history of malignant tumors } \\
\hline Yes & 3 & 1 & 2 & \multirow[t]{2}{*}{0.460} \\
\hline No & 26 & 8 & 18 & \\
\hline \multicolumn{5}{|l|}{ P16 expression } \\
\hline Positive & 29 & 9 & 20 & \\
\hline Negative & 0 & 0 & 0 & \\
\hline \multicolumn{5}{|l|}{ Primary tumor size(cm) } \\
\hline$<3$ & 7 & 5 & 2 & \multirow[t]{2}{*}{$0.015^{*}$} \\
\hline$\geq 3$ & 22 & 4 & 18 & \\
\hline \multicolumn{5}{|c|}{ Pelvic lymph node metastasis } \\
\hline Positive & 8 & 2 & 6 & \multirow[t]{2}{*}{0.325} \\
\hline Negative & 21 & 7 & 14 & \\
\hline \multicolumn{5}{|l|}{ Infiltration depth } \\
\hline $\begin{array}{l}\text { Superficial/Deep fibrous } \\
\text { histiocytoma }\end{array}$ & 18 & 8 & 10 & \multirow[t]{2}{*}{$0.048^{*}$} \\
\hline Outer layer of the cervix & 11 & 1 & 10 & \\
\hline \multicolumn{5}{|l|}{ The FIGO stage } \\
\hline$|a-| b \mid$ & 15 & 8 & 7 & \multirow[t]{2}{*}{$0.009^{*}$} \\
\hline |b2-1|a2 & 14 & 1 & 13 & \\
\hline
\end{tabular}

${ }^{*} \mathrm{P}<0.05$

FIGO International Federation of Gynecology and Obstetrics

Of course, in view of total 53 patient samples (24 highgrade squamous intraepithelial lesion tissues and 29 cervical squamous cell cancer tissues), a large numbers of patients will contribute to understand the correlation between HMGB2 expression and clinicopathological parameters of cervical cancer patients better. Additionally, it should be noticed that the different cellularity of normal cervical tissues, high-grade squamous intraepithelial lesion tissues and cervical squamous cell cancer tissues might be an interference factor during the analysis.

In conclusion, notwithstanding these limitations, we identified 72 differentially expressed proteins in cervical cancer samples compared to normal cervical samples and high-grade squamous intraepithelial lesion samples. Most of the identified proteins were nucleic acid binding proteins. Moreover, further research results showed that HMGB2 was frequently up-regulated in cervical cancer samples and its up-regulation was associated with primary tumor size, infiltration depth and FIGO stage, resulting in tumor progression. These results suggest that HMGB2 may contribute to the progression of cervical cancer and that the presence of HMGB2 in cervical cancer tissues may be a prognostic indicator for cervical cancer patients.

\section{Supplementary Information}

The online version contains supplementary material available at https://doi. org/10.1186/s12014-020-09308-4.

Additional file 1: Table S1. The HPV information of the samples. Figure

S1. The representative microphotographs of IRS score 1, 2 and 3 for HMGB2.

\section{Acknowledgements}

Not applicable.

\section{Authors' contributions}

$\mathrm{XH}$ : Methodology, data collection \& analysis, manuscript writing; SYZ: Methodology, data collection \& analysis, Writing — review \& editing; PNZ: Methodology, Writing — review \& editing; YML: Data collection \& analysis, Writing — review \& editing; SSS: Providing samples and Data collection, Writing —review \& editing, CQW: Providing samples, Writing — review \& editing; SJG: Conceptualization, project administration, Supervision, Writing - review \& editing. All authors read and approved the final manuscript.

\section{Funding}

This work was supported by the Natural Science Fund of Shanghai Science and Technology Committee (Grant No. 17ZR1403400)

Availability of data and materials

All data generated or analyzed during this study are included in this published article.

\section{Ethics approval and consent to participate}

All procedures performed in studies involving human participants were in accordance with the ethical standards of the review board and ethics committee of Obstetrics and Gynecology Hospital affiliated with Fudan University and with the 1964 Helsinki declaration and its later amendments or comparable ethical standards. Informed consent was obtained from all individual participants included in the study.

\section{Consent for publication}

All authors agree to be published.

\section{Competing interests}

The authors declare that they have no competing interests.

\section{Author details}

${ }^{1}$ Center of Diagnosis and Treatment for Cervical Diseases, Obstetrics and Gynecology Hospital of Fudan University, No. 419, Fangxie Road, Huangpu District, Shanghai 200011, China. ${ }^{2}$ Shanghai Key Laboratory of Female Reproductive Endocrine-Related Disease, Fudan University, Shanghai 200011, China. ${ }^{3}$ Department of Gynecology, Obstetrics and Gynecology Hospital of Fudan University, Shanghai 200011, China.

Received: 29 August 2020 Accepted: 7 December 2020

Published online: 06 January 2021

References

1. Paradkar PH, Joshi JV, Mertia PN, Agashe SV, Vaidya RA. Role of cytokines in genesis, progression and prognosis of cervical cancer. Asian Pac J Cancer Prev. 2014;15(9):3851-64.

2. Wenzel L, Dogan-Ates A, Habbal R, et al. Defining and measuring reproductive concerns of female cancer survivors. J Natl Cancer Inst Monogr. 2005. https://doi.org/10.1093/jncimonographs/lgi017(34),94-98. 
3. Del Campo JM, Prat A, Gil-Moreno A, Perez J, Parera M. Update on novel therapeutic agents for cervical cancer. Gynecol Oncol. 2008;110(3 Suppl 2):S72-76.

4. Luvero D, Plotti F, Lopez S, et al. Antiangiogenics and immunotherapies in cervical cancer: an update and future's view. Med Oncol. 2017;34(6):115.

5. Yoo MW, Park J, Han HS, et al. Discovery of gastric cancer specific biomarkers by the application of serum proteomics. Proteomics. 2017. https ://doi.org/10.1002/pmic.201600332.

6. Swiatly A, Horala A, Matysiak J, Hajduk J, Nowak-Markwitz E, Kokot ZJ. Understanding ovarian cancer: iTRAQ-based proteomics for biomarker discovery. Int J Mol Sci. 2018;19(8):2240.

7. She $S$, Jiang $L$, Zhang $Z$, et al. Identification of the C-reactive protein interaction network using a bioinformatics approach provides insights into the molecular pathogenesis of hepatocellular carcinoma. Cell Physiol Biochem . 2018;48(2):741-52.

8. Boichenko AP, Govorukhina N, Klip HG, et al. A panel of regulated proteins in serum from patients with cervical intraepithelial neoplasia and cervical cancer. J Proteome Res. 2014;13(11):4995-5007.

9. Panis C, Pizzatti L, Abdelhay E. How can proteomics reach cancer biomarkers? Curr Proteomics. 2013;10(2):136-49.

10. Syed N, Chavan S, Sahasrabuddhe NA, et al. Silencing of high-mobility group box 2 (HMGB2) modulates cisplatin and 5-fluorouracil sensitivity in head and neck squamous cell carcinoma. Proteomics. 2015;15(2-3):383-93.

11. Shadforth IP, Dunkley TP, Lilley KS, Bessant C. i-Tracker: for quantitative proteomics using iTRAQ. BMC Genomics. 2005;6:145.

12. Da Huang W, Sherman BT, Lempicki RA. Systematic and integrative analysis of large gene lists using DAVID bioinformatics resources. Nat Protoc. 2009;4(1):44-57.

13. Da Huang W, Sherman BT, Lempicki RA. Bioinformatics enrichment tools: paths toward the comprehensive functional analysis of large gene lists. Nucleic Acids Res. 2009;37(1):1-13.

14. Mi H, Huang $X$, Muruganujan A, et al. PANTHER version 11: expanded annotation data from Gene Ontology and Reactome pathways, and data analysis tool enhancements. Nucleic Acids Res. 2017;45(D1):D183-9.

15. Bindea G, Mlecnik B, Hackl H, et al. ClueGO: a Cytoscape plug-in to decipher functionally grouped gene ontology and pathway annotation networks. Bioinformatics. 2009;25(8):1091-3.

16. Bindea G, Galon J, Mlecnik B. CluePedia Cytoscape plugin: pathway insights using integrated experimental and in silico data. Bioinformatics. 2013;29(5):661-3.

17. Pallier C, Scaffidi P, Chopineau-Proust S, et al. Association of chromatin proteins high mobility group box (HMGB) 1 and HMGB2 with mitotic chromosomes. Mol Biol Cell. 2003;14(8):3414-26.

18. Ronfani L, Ferraguti M, Croci L, et al. Reduced fertility and spermatogenesis defects in mice lacking chromosomal protein Hmgb2. Development. 2001;128(8):1265-73.

19. Zheng S, Weng Q, Wu W, Ding G. Blood purification treatment initiated at the time of sepsis diagnosis effectively attenuates serum HMGB1 upregulation and improves patient prognosis. Exp Ther Med. 2017; 14(4):3029-35.

20. Li YB, Xu P, Xu K, et al. Methotrexate affects HMGB1 expression in rheumatoid arthritis, and the downregulation of HMGB1 prevents rheumatoid arthritis progression. Mol Cell Biochem. 2016;420(1-2):161-70.

21. Cheng $\mathrm{H}$, Wang W, Zhang $\mathrm{Y}$, et al. Expression levels and clinical significance of hepsin and HMGB1 proteins in cervical carcinoma. Oncol Lett. 2017;14(1):159-64.

22. Chung HW, Lim JB, Jang S, Lee KJ, Park KH, Song SY. Serum high mobility group box-1 is a powerful diagnostic and prognostic biomarker for pancreatic ductal adenocarcinoma. Cancer Sci. 2012;103(9):1714-21.

23. Zhao CB, Bao JM, Lu YJ, et al. Co-expression of RAGE and HMGB1 is associated with cancer progression and poor patient outcome of prostate cancer. Am J Cancer Res. 2014;4(4):369-77.
24. Exner R, Sachet M, Arnold T, et al. Prognostic value of HMGB1 in early breast cancer patients under neoadjuvant chemotherapy. Cancer Med. 2016;5(9):2350-8.

25. Ladoire S, Enot D, Senovilla L, et al. The presence of LC3B puncta and HMGB1 expression in malignant cells correlate with the immune infiltrate in breast cancer. Autophagy. 2016;12(5):864-75.

26. Xie B, Cao K, Li J, et al. Hmgb1 inhibits Klotho expression and malignant phenotype in melanoma cells by activating NF-kappaB. Oncotarget. 2016;7(49):80765-82.

27. Wen S, Du X, Gou Y, Jiang L. Treatment effects of oxaliplatin combined with gemcitabine on colorectal cancer and its influence on HMGB1 expression. Oncol Lett. 2016;12(5):3187-90.

28. Tang L, Chai W, Ye F, et al. HMGB1 promotes differentiation syndrome by inducing hyperinflammation via MEK/ERK signaling in acute promyelocytic leukemia cells. Oncotarget. 2017;8(16):27314-27.

29. Pasheva EA, Pashev IG, Favre A. Preferential binding of high mobility group 1 protein to UV-damaged. DNA Role of the $\mathrm{COOH}$-terminal domain. J Biol Chem. 1998;273(38):24730-6.

30. Huang JC, Zamble DB, Reardon JT, Lippard SJ, Sancar A. HMG-domain proteins specifically inhibit the repair of the major DNA adduct of the anticancer drug cisplatin by human excision nuclease. Proc Natl Acad Sci U S A. 1994;91(22):10394-8.

31. He SJ, Cheng J, Feng X, Yu Y, Tian L, Huang Q. The dual role and therapeutic potential of high-mobility group box 1 in cancer. Oncotarget. 2017;8(38):64534-50.

32. Hao Q, Du XQ, Fu X, Tian J. Expression and clinical significance of HMGB1 and RAGE in cervical squamous cell carcinoma. Zhonghua zhong liu za zhi [Chinese J Oncol]. 2008;30(4):292-5.

33. Koon N, Schneider-Stock R, Sarlomo-Rikala M, et al. Molecular targets for tumour progression in gastrointestinal stromal tumours. Gut. 2004;53(2):235-40.

34. Guggenheim ER, Xu D, Zhang CX, Chang PV, Lippard SJ. Photoaffinity isolation and identification of proteins in cancer cell extracts that bind to platinum-modified DNA. Chembiochem : a European journal of chemical biology. 2009;10(1):141-57.

35. Balani P, Boulaire J, Zhao Y, Zeng J, Lin J, Wang S. High mobility group box2 promoter-controlled suicide gene expression enables targeted glioblastoma treatment. Mol Ther . 2009;17(6):1003-11.

36. Kwon JH, Kim J, Park JY, et al. Overexpression of high-mobility group box 2 is associated with tumor aggressiveness and prognosis of hepatocellular carcinoma. Clin Cancer Res . 2010;16(22):5511-21.

37. Cai $X$, Ding H, Liu $Y$, et al. Expression of HMGB2 indicates worse survival of patients and is required for the maintenance of Warburg effect in pancreatic cancer. Acta Biochim Biophys Sin. 2017;49(2):119-27.

38. Wang LL, Meng QH, Jiao Y, et al. High-mobility group boxes mediate cell proliferation and radiosensitivity via retinoblastoma-interactiondependent and -independent mechanisms. Cancer Biother Radiopharm . 2012;27(5):329-35.

39. Zhang P, Lu Y, Gao S. High-mobility group box 2 promoted proliferation of cervical cancer cells by activating AKT signaling pathway. J Cell Biochem. 2019;120(10):17345-53.

40. Fu D, Li J, Wei J, et al. HMGB2 is associated with malignancy and regulates Warburg effect by targeting LDHB and FBP1 in breast cancer. Cell Commun Signal. 2018;16(1):8.

41. Redmond AM, Byrne C, Bane FT, et al. Genomic interaction between ER and HMGB2 identifies DDX18 as a novel driver of endocrine resistance in breast cancer cells. Oncogene. 2015;34(29):3871-80.

\section{Publisher's Note}

Springer Nature remains neutral with regard to jurisdictional claims in published maps and institutional affiliations. 